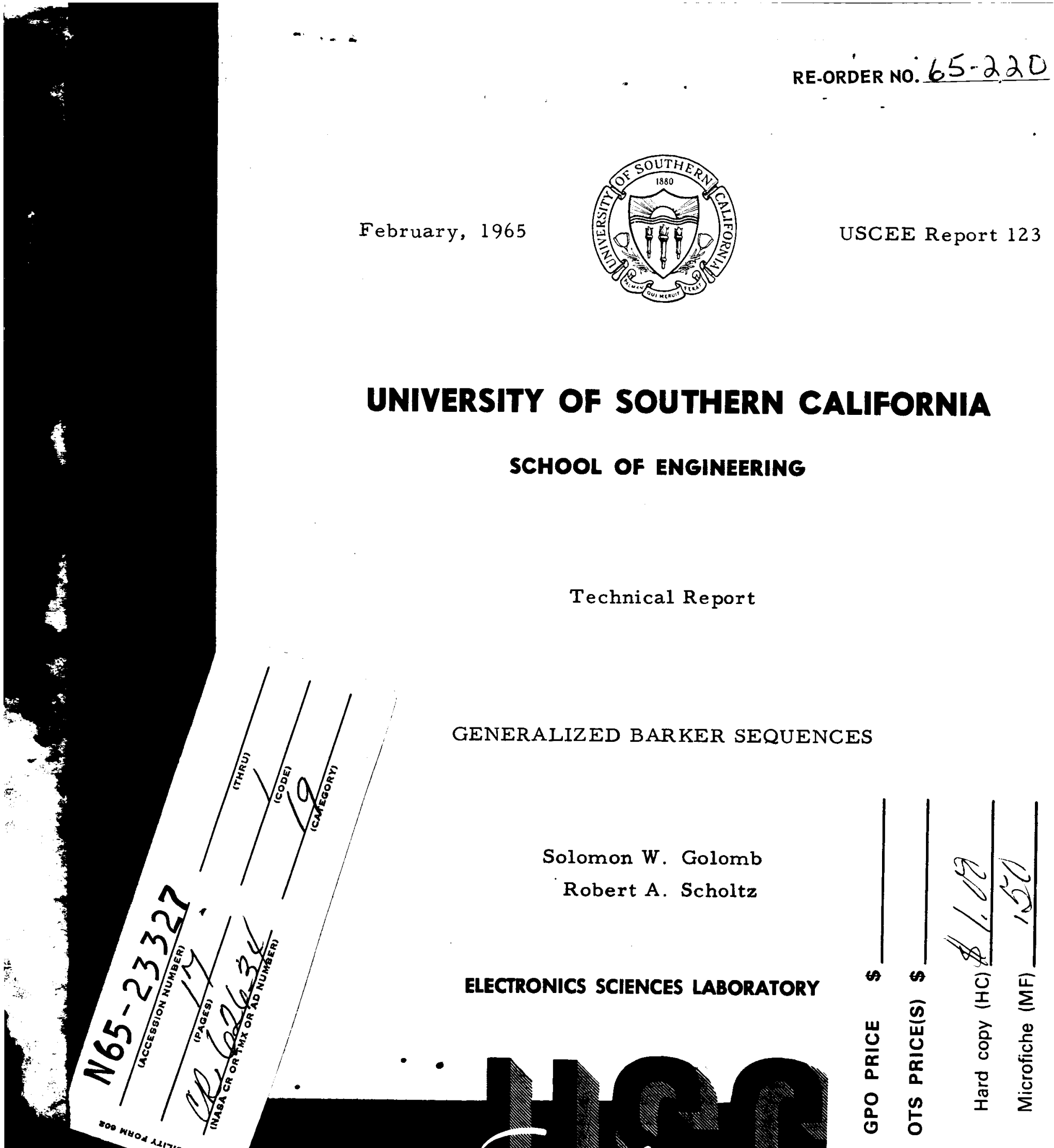


USCEE 123

GENERALIZED BARKER SEQUENCES

Technical Report

Is sued By

Electronic Sciences Laboratory

University of Southern California

Los Angeles, California

February 1965

Solomon W. Golomb

Robert A. Scholtz

This work was performed for the Jet Propulsion Laboratory, California Institute of Technology, sponsored by the National Aeronautics and Space Administration under Contract NAS7-100.

\section{ACKNOW LEDGEMENT}

Prepared by the Electronic Sciences Laboratory, University of Southern California

Supported by the Jet Propulsion Laboratory/NASA,

Under contract no. 951076 


\section{ABSTRACT}

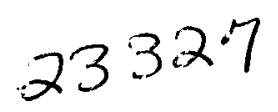

A generalized Barker sequence is a finite sequence $\left\{a_{r}\right\}$ of complex numbers having absolute value 1 , and possessing a correlation function $C(\tau)$ satisfying the constraint $|C(\tau)| \leq 1, \tau \neq 0$. Classes of transformations leaving $|C(\tau)|$ invariant are exhibited. Constructions for generalized Barker sequences of various lengths and alphabet sizes are given. Sextic Barker sequences are investigated and examples given for all lengths through thirteen. No theoretical limit to the length of sextic sequences has been found. 


\section{TABLE OF CONTENTS}

Page

Abstract i

1. Introduction 1

2. Barker-Preserving Transformations 2

3. Specific Examples 3

4. General Alphabets 6

5. The Sextic Alphabet 9

6. Sequences of Length $7 \quad 11$

References 13 


\section{GENERALIZED BARKER SEQUENCES}

Solomon W. Golomb

Robert A. Scholtz

1. Introduction

A Barker sequence [l] is a sequence $\left\{a_{r}\right\}$ of $+l^{\prime}$ 's and -1 's of some finite length $k$ such that the correlation function $C(\tau)$ defined by $C(\tau)=\sum_{r=1}^{k-\tau} a_{r} a_{r+\tau}^{*}$ satisfies $C(\tau) \leqq 1$ for $\tau \neq 0$. In the present note, consider "generalized Barker sequences" $\left\{a_{r}\right\}$ of finite length $k$, where the terms $a_{r}$ are allowed to be complex numbers of absolute value 1 , where correlation is now the Hermitian dot product $C(\tau)=\sum_{r=1}^{k-\tau} a_{r} a_{r+\tau}^{*}$ $\left(z^{*}\right.$ denotes the complex conjugate of $z$ ), and the same restriction $C(\tau) \leqq 1$ for $\tau \neq 0$ is imposed. We will exhibit a class of transformations which leave the absolute value of the correlation function unaltered, so that, in particular, generalized Barker sequences are changed into other generalized Barker sequences. We examine the effect of these transformations on the original $( \pm 1)$ Barker sequences and on four valued $( \pm 1, \pm i)$ Barker sequences; and then exhibit constructions for generalized Barker sequences of a variety of lengths, and all possible alphabet sizes. Finally, we observe some very interesting facts about sextic Barker sequences, including that they occur for all lengths 
tested (i.e. for $k \leqq 13$ ), and could conceivably occur for all possible sequence lengths.

\section{Barker-Preserving Transformations}

Let $\left\{u_{r}\right\}$ be a complex-valued sequence of length $k$, and let its auto-correlation function $C_{u}(\tau)=\sum_{r=1}^{k-\tau} u_{r} u_{r+T}^{*}$. We define a new complexvalued sequence $\left\{\mathrm{v}_{\mathrm{r}}\right\}$ of length $\mathrm{k}$ as follows:

$$
v_{r}=u_{r} e^{\frac{2 \pi i r}{m}}
$$

where $\mathrm{m}$ is any non-zero integer. Then we observe that the auto-correlation function $\mathrm{C}_{\mathrm{v}}(\tau)$ of $\left\{\mathrm{v}_{\mathbf{r}}\right\}$ satisfies

$$
C_{v}(\tau)=\sum_{r=1}^{k-\tau} v_{r} v_{r+\tau}^{*}=\sum_{r=1}^{k-\tau} u_{r} e^{\frac{2 \pi i r}{m}} u_{r+\tau}^{*} e^{\frac{-2 \pi i(r+\tau)}{m}}
$$

$$
=\sum_{r=1}^{k-\tau} u_{r} u_{r+\tau}^{*} e^{\frac{-2 \pi i \tau}{m}}=e^{\frac{-2 \pi i \tau}{m}} C_{u}(\tau)
$$

for all $\tau$. In particular, since $\left|e^{\frac{-2 \pi i \tau}{m}}\right|=1$, we have $\left|C_{V}(\tau)\right|=\left|C_{u}(\tau)\right|$ for all $\tau$, as well as $\left|v_{\mathbf{r}}\right|=\left|u_{r}\right|$ by virtue of equation (1). As a special case, if $\left|u_{r}\right|=1$ for all $r$, the transformation (1) takes a 
generalized Barker sequence of length $k$ into another generalized Barker sequence of length $\mathrm{k}$.

Further we observe that the somewhat more general transforma-

tion

$$
v_{r}=u_{r} e^{\frac{2 \pi i(r+\alpha)}{x}}
$$

where $\alpha$ and $x$ are any real numbers, $\mathbf{x} \neq 0$, also preserves $\left|v_{r}\right|=\left|u_{r}\right|$ and $\left|\mathrm{C}_{\mathbf{v}}(\tau)\right|=\left|\mathrm{C}_{\mathbf{u}}(\tau)\right|$. Finally, the transformation $\mathrm{v}_{\mathbf{r}}=u_{\mathrm{k}-\mathrm{r}+\mathrm{l}}$, which merely runs the sequence backwards, clearly preserves the Barker property, as does the transformation $w_{r}=u_{r}^{*}$ (where the star again denotes complex conjugation).

In summary, there is a group of $4 \mathrm{~m}^{2}$ transformations, each of which takes $m$-phase Barker sequences into $m$-phase Barker sequences. As generators of this group, we may take the two order -2 transformations of time reversal and complex conjugation, and the two order $-\mathrm{m}$ transformations of constant multiplication by $\zeta$ and progressive

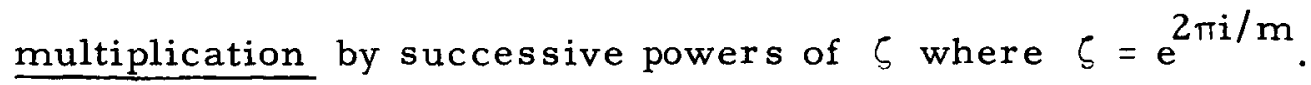

\section{Specific Examples}

If we take $m=2$ in equation (1), then the sequence $\left(u_{1}, u_{2}, u_{3}, u_{4}, \ldots, u_{k}\right)$ is changed into $\left(u_{1},-u_{2}, u_{3}-u_{4} \ldots,(-1)^{k-1} u_{k}\right)$. In particular, this transformation changes ordinary binary Barker 
sequences into other such sequences, though nothing very startling is obtained in this way. Taking $m=4$, the sequence $\left(u_{1}, u_{2}, u_{3}, u_{4} \ldots, u_{k}\right)$ is changed into $\left(u_{1}, i u_{2}-u_{3},-i u_{4}, \ldots, i^{k-1} u_{k}\right)$. In particular, this transformation sets up a one-to-one correspondence between ordinary (binary) Barker sequences and four-symbol Barker sequences (the symbols being $\pm 1, \pm i)$ in which real and imaginary terms alternate. This correspondence is illustrated in Table I for binary Barker sequences of all known lengths.

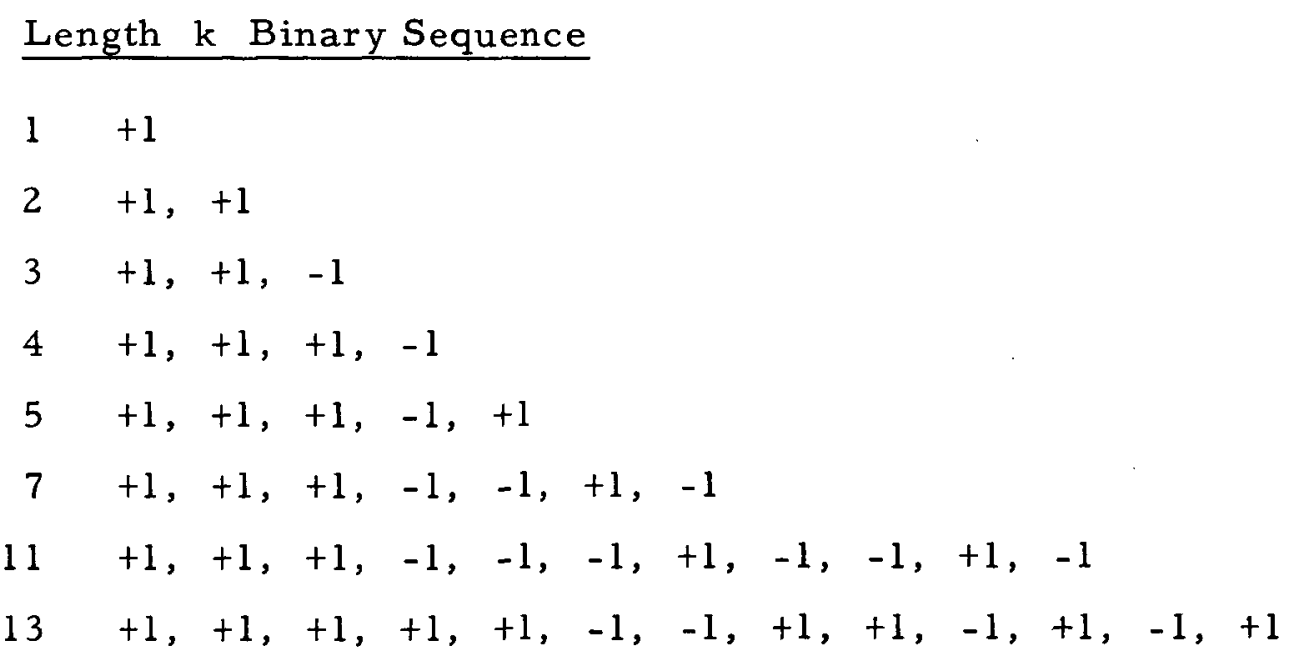

\section{Quaternary Barker Sequence}

$$
\begin{aligned}
& 1+1 \\
& 2+1,+\mathbf{i} \\
& 3+1,+i,+1 \\
& 4+1,+\mathrm{i},-1,+\mathrm{i} \\
& 5+1 .+i,-1,+i,+1 \\
& 7+1,+i,-1,+i,-1,+i,+1 \\
& 11+1,+i,-1,+i,-1,-i,-1,+i,-1,+i,+1 \\
& 13+1,+i,-1,-i,+1,-i,+1,-i,+1,-i,-1,+i,+1
\end{aligned}
$$

TABLE I: Correspondence between binary and alternating quarternary Barker Sequences. 
(By the results of Storer and Turyn [2], there are no other binary Barker sequences of odd length and the possibility of other such sequences of even length appears quite remote.) Another description of the correspondence is that between successive terms in the binary sequence, there is a transition of either $0^{\circ}$ or $180^{\circ}$, while between successive terms in the alternating quaternary sequence, there is a transition of either $90^{\circ}$ or $270^{\circ}$, and we let $0^{\circ}$ transitions correspond to $90^{\circ}$ transitions, while $180^{\circ}$ transitions correspond to $270^{\circ}$ transitions, respectively.

It is interesting to note that the quaternary alternating Barker sequences of odd length are all palindromic (i.e., read the same forward and backward), thus evidencing a symmetry which is obscured in the binary representation.

Next, we consider a quaternary Barker sequence of length 15 [3] which is not equivalent to a binary Barker sequence. In Table II, we see such a sequence, together with its transforms corresponding to $m=2$ and to $m=4$.

Original Sequence $\quad+1,-1,+1,+i,-i,-1,+i,-i,-i,+1,+i,+i,+1,+1,+1$ Transform with $m=2+1,+1,+1,-i,-i,+1,+i,+i,-i,-1,+i,-i,+1,-1,+1$ Transform with $\mathrm{m}=4+1,-i,-1,+1,-i,-i,-i,-1,-i,+i,-i,+1,+1,+i,-1$

TABLE II. Transformations on a Quaternary Barker Sequence 
We observe that the effect of the $m=2$ transformation is to replace the original terms by their complex conjugates in reverse order. Since reversal of order and conjugation of elements are both elementary symmetries which preserve Barker correlation, nothing essentially new has been obtained. However, the transformation with $m=4$ yields a very different sequence from the original. For example, whereas the original sequence had one run of length 3 and two runs of length 2 each, the transformed sequence has one run of length 3 but only one run of length 2. Also the original sequence has its run of length 3 at the end, whereas the transformed sequence has its longest run near the middle.

It may be mentioned in passing that despite its title, Reference [4] contains no examples of quaternary Barker codes.

\section{General Alphabets}

Let $\rho=e^{2 \pi i / n}$, and define the alphabet $A_{n}$ to be the set $\left\{1, \rho, \rho^{2}, \rho^{3}, \ldots, \rho^{n-1}\right\}$. For subsequent convenience, we also define $\eta=\rho^{[(n+1) / 2]}$, the lowest power of $\rho$ whose phase angle is at least $180^{\circ}$.

We ask the general question on the existence of Barker sequences as follows: "For given sequence length $k$, what are all the values of $n$ such that a Barker sequence of length $k$ can be constructed from the 
alphabet $A_{n}$ ?"

In Table III, we answer this question completely for $k=1,2,3,4,5$ by exhibiting constructions which work for all $\mathrm{n} \geqq 2$. A couple of simple observations will assist the reader in verifying that the out-of-phase correlations are indeed $\leqq 1$, as required:

\begin{tabular}{l|l|l|l}
$\mathrm{k}$ & \multicolumn{1}{|c|}{ sequence } & \multicolumn{1}{|c}{ correlation function } & \multicolumn{1}{|c}{ which $\mathrm{n} ?$} \\
\hline 1 & 1 & 1 & all $\mathrm{n} \geq 1$ \\
2 & 1,1 & 2,1 & all $\mathrm{n} \geq 1$ \\
3 & $1,1, \eta$ & $3,1+\eta, \eta$ & all $\mathrm{n}=2$ \\
4 & $1,1, \eta, 1$ & $4,1+\eta+\eta^{*}, 1+\eta, 1$ & all $\mathrm{n} \geq 2$ \\
5 & $1,1,1,-1,1$ & $5,0,1,0,1$ & all even $\mathrm{all} n \geq 2$ \\
& $1,1, \rho, \eta, \rho$ & $5,1+\rho+\eta+\eta^{*}, 1+\rho+\eta, \rho+\eta, \rho$ & all except $1,2,4 \quad$
\end{tabular}

TABLE III. Construction for generalized Barker sequences for all lengths up to 5, and all alphabet sizes greater than 1.

a) The sum of 2 unit vectors lies within the unit circle if and only if the angle between those vectors is between $120^{\circ}$ and $240^{\circ}$. (In particular, $1+\eta$ lies within or on the unit circle for $n \geq 2$, the case $n=3$ being the tightest squeeze, while $\rho+\eta$ lies within or on the unit circle except when $\mathrm{n}=1,2,4$, the cases $\mathrm{n}=3$ and 6 being tightest.)

b) The sum of 3 unit vectors lies within the unit circle if and only if there is no semi-circle properly containing all three vectors. (In particular, since the sets $1, \eta, \eta^{*}$ and $1, \rho, \eta$ each defy containment within a 
semicircle, the vectors $1+\eta+\eta^{*}$ and $1+\rho+\eta$ do not extend beyond the unit circle.) Finally, we may observe that $1+\rho+\eta+\eta^{*}$ lies outside the unit circle for $n=1,2,4$; on the unit circle for $n=3,5,6$; and within the unit circle for $n \geq 7$. (In fact, this vector sum decreases in magnitude to zero as $\mathrm{n}$ increases to infinity.)

A simple necessary and sufficient condition for the sum of four unit vectors to lie within the unit circle has not been found. However, the following two necessary conditions are sharp:

a) The four vectors must not be contained within an arc of less than $2 \cos ^{-1}\left(\frac{1}{4}\right) \approx 151^{\circ}$.

b) No three of the four vectors may be contained within an arc of less than $180^{\circ}-\cos ^{-1}\left(\frac{1}{4}\right) \approx 104.5^{\circ}$.

For the case $k=6$, it has long been known that there is no binary Barker sequence. An exhaustive search has shown that there is also not generalized Barker sequence of length 6 for alphabet sizes of 3, 4, or 5. However, the following example using the alphabet $A_{6}$ has been found:

$$
1,1, \epsilon,-1,1,-\epsilon
$$

where $\epsilon=e^{2 \pi i / 6}$. The correlation values for this sequence are 6. $\epsilon^{2}, \epsilon^{1}, \epsilon^{3}, \epsilon^{5}, \epsilon^{4}$. 
Except for the Barker-preserving transformations of Section 2, this example is unique. However, in one of its transformed avatars, it takes the form

$$
1, \epsilon,-1,1,-\epsilon,-1
$$

for which the correlation values are $6,-1,-1,1,-1,-1$.

A Barker sequence is called palindromic if the sequence reads the same forwards and backwards, except for at most a real constant factor. Clearly the correlation function of a palindromic sequence is real. We have observed that there are sextic palindromic sequences of every length through 9, but apparently none of length 10 .

\section{The Sextic Alphabet}

There is strong evidence to indicate that a Barker sequence of length 6 can only be achieved over an alphabet $A_{n}$ for which $n$ is a multiple of 6. Moreover, the sixth roots of unity (with zero adjoined) have remarkable arithmetic which is partially closed under addition, as well as closed under multiplication.

In Table IV, we see the present state of knowledge concerning binary, ternary, quaternary, and sextic Barker sequences. The appropriate earlier references for these cases are: Binary [2], 
Ternary [5], and Quaternary [3]. However, these cases have all been rechecked. In Table IV a blank indicates that no such sequence exists, while a question mark means that the question has not yet been exhaustively searched.

\begin{tabular}{r|r|r|r|l|}
$\mathrm{n}$ & 2 & 3 & 4 & 6 \\
\hline 1 & $\checkmark$ & $\checkmark$ & $\checkmark$ & $\checkmark$ \\
\hline 2 & $\checkmark$ & $\checkmark$ & $\checkmark$ & $\checkmark$ \\
\hline 3 & $\checkmark$ & $\checkmark$ & $\checkmark$ & $\checkmark$ \\
\hline 4 & $\checkmark$ & $\checkmark$ & $\checkmark$ & $\checkmark$ \\
\hline 5 & $\checkmark$ & $\checkmark$ & $\checkmark$ & $\checkmark$ \\
\hline 6 & & & & $\checkmark$ \\
\hline 7 & $\checkmark$ & $\checkmark$ & $\checkmark$ & $\checkmark$ \\
\hline 8 & & & & $\checkmark$ \\
\hline 9 & & $\checkmark$ & & $\checkmark$ \\
\hline 10 & & & & $\checkmark$ \\
\hline 11 & $\checkmark$ & & $\checkmark$ & $\checkmark$ \\
\hline 12 & & & & $\checkmark$ \\
\hline 13 & $\checkmark$ & & $\checkmark$ & $\checkmark$ \\
\hline 14 & & & & $?$ \\
\hline 15 & & & $\checkmark$ & $?$ \\
\hline
\end{tabular}

TABLE IV: Comparison of the sequences available from the alphabet $A_{2}, A_{3}, A_{4}$, and $A_{6}$.

We see that for lengths $k=6,8,10$, and 12, there are no examples of binary, ternary, or quaternary sequences, but that sextic examples exist, as follows: 


$$
\begin{aligned}
& S_{6}=1,1, \epsilon,-1,1,-\epsilon \\
& S_{8}=1,1, \epsilon, 1,-\epsilon, \epsilon,-1, \epsilon^{*} \\
& S_{10}=1,1,-\epsilon,-1,-\epsilon^{*},-\epsilon,-1, \epsilon^{*},-\epsilon^{*},-\epsilon \\
& S_{12}=1,1, \epsilon, 1, \epsilon^{*},-\epsilon^{*},-\epsilon^{*},-\epsilon, 1,-\epsilon, \epsilon,-1
\end{aligned}
$$

Examples of length 6, 8, and 10 were first discovered by the authors. Examples of length 12 were found in a computer search conducted by Harold Fredricksen. Of these, only $\mathrm{S}_{6}$ is unique up to Barkerpreserving transformations. This is one of several facts supporting the highly tentative conjecture that sextic Barker sequences exist for all sequence lengths, in sharp contrast to the binary case [2]. That is, the search routines indicate that sextic examples may become more numerous as the sequence length increases.

\begin{tabular}{|c|c|c|c|}
\hline $\mathbf{k}$ & sequence & correlation function & which $\mathrm{n}$ ? \\
\hline 7 & $\begin{array}{l}1,1,1,-1,-1,1,-1 \\
1,1, \beta, \beta, 1, \beta, 1\end{array}$ & $\begin{array}{l}7,0,-1,0,-1,0,-1 \\
7,2(1+u), 2+u+\beta, \\
2+u, 1+u, 1+\beta, 1 \\
\text { where } u=\beta+\beta^{*} .\end{array}$ & $\begin{array}{l}\text { all even } n \\
\text { all odd } n>1 \\
\text { except } 5 \& 7 \text {, as well } \\
\text { as large even } n \text {. }\end{array}$ \\
\hline
\end{tabular}

\section{Sequences of Length 7}

The case of sequence length 7 has been settled definitively, as shown in Table $\mathrm{V}$, which augments Table III.

TABLE V 
Here $\beta$ is an element of $A_{n}$ with a phase angle between $120^{\circ}$ and $\pi-\cos ^{-1}(3 / 4) \approx 138 \frac{1}{2}^{\circ}$. Not only are there no $5^{\text {th }}$ or $7^{\text {th }}$ roots of unity with phase angles in this range, but an exhaustive search has shown that there are no Barker sequences of length 7 over $A_{5}$ or $A_{7}$. 


\section{REFERENCES}

1. Barker, R.H., Group Synchronizing of Binary Digital Systems, Communication Theory, London, 1953, pp. 273-287.

2. Turyn, R. and Storer, J., On Binary Sequences, Proceedings of the American Mathematical Society, Vol. 12, No. 3, June, 1961, pp. $394-399$.

3. Carley, G., Unpublished report.

4. Welti, G.R. Quaternary Codes for Pulsed Radar, IRE Transaction on Information Theory, IT-6, Number 3, June, 1960.

5. de Long, D.F. Jr., Three-Phase Codes, MIT Lincoln Laboratory, Lexington, Massachusetts, Group Report. $47-28$, July 24, 1959. 